\title{
Comparación de la salud subjetiva entre prototipos de personalidad recuperados en población general de México
}

\author{
Pedro Solís-Cámara ${ }^{1 *}$, Rosa Martha Meda Lara ${ }^{2}$, Bernardo Moreno Jiménez ${ }^{3}$, Andrés Palomera Chávez ${ }^{2}$ \\ y Pedro Juárez Rodríguez ${ }^{2}$ \\ ${ }^{1}$ Centro Universitario de la Ciénega, Universidad de Guadalajara, ${ }^{2}$ Centro Universitario de Ciencias de la Salud, \\ Universidad de Guadalajara, ${ }^{3}$ Facultad de Psicología, Universidad Autónoma de Madrid
}

Recibido, agosto 29/2016

Concepto de evaluación, enero 16/2017

Aceptado, mayo 5/2017

\begin{abstract}
Referencia: Solís-Cámara R., P., Meda Lara, R.M., Moreno Jiménez, B., Palomera Chávez, A. \& Juárez Rodríguez, P. (2017). Comparación de la salud subjetiva entre prototipos de personalidad recuperados en población general de México. Acta colombiana de Psicología, 20(2), 200-213. doi: http://www.dx.doi.org/10.14718/ACP.2017.20.2.10
\end{abstract}

Resumen

El objetivo de este estudio fue obtener prototipos de personalidad en población general de México y comparar la salud subjetiva entre ellos. En total, participaron 994 individuos de 14 a 63 años de edad. Se evaluaron cinco rasgos de personalidad (Neuroticismo, Extraversión, Apertura, Responsabilidad y Amabilidad) con el NEO-FFI, y la salud subjetiva con el GHQ-12 y una pregunta sobre el estado de salud. Para corroborar la consistencia de los prototipos se dividió la muestra en dos grupos de edad: joven (de 14 a 25 años) y maduro (de 26 a 63 años). Y como resultado se recuperaron tres prototipos en ambos grupos: las personas Resilientes — bajo neuroticismo y alto en el resto de los rasgos_-, quienes tuvieron la mejor salud subjetiva; las No-Resilientes — alto neuroticismo y bajo en el resto de los rasgos_-, que presentaron la peor salud subjetiva; y las personas Disciplinadas — alto en responsabilidad y promedio en los otros rasgos-, que presentaron una salud subjetiva intermedia en comparación con los otros prototipos. Finalmente, la autodisciplina y la resiliencia fueron los rasgos que discriminaron mejor la buena salud. Los hallazgos se discuten en términos de la generalización de prototipos a través de culturas.

Palabras clave: Disciplinado, no-resiliente, personalidad, prototipos, resiliente, salud.

\section{Comparison of subjective bealth between personality prototypes extracted from general population of Mexico}

\begin{abstract}
The objective of this study was to extract personality prototypes from general population of Mexico and to compare subjective health indicators between these prototypes. Participants were 994 individuals (aged 14 to 63 years). Five personality traits (Neuroticism, Extraversion, Openness, Conscientiousness, and Agreeableness) were assessed with the NEO-FFI. Subjective indicators of health (self-rated health and psychological distress) were assessed with a question regarding health status and the GHQ-12. To verify the consistency of the prototypes, the sample was divided into two age groups, young (14 to 25 years) and mature (26 to 63 years.). Three stable prototypes were recovered from both groups. Resilient individuals (low neuroticism and high in other traits) had the best subjective health; the Non-Resilient individuals (high neuroticism and low in other traits) had the worst subjective health; and Self-Disciplined individuals (high conscientiousness and medium scores in other traits) were in the middle of these extremes in subjective health. Self-discipline and resilience were most discriminative in terms of subjective health. Findings are discussed in terms of the generalization of prototypes across cultures.

Key words: Self disciplined, non-resilient, personality, prototypes, resilient, health.
\end{abstract}

* Av. Universidad \#1115. CP 47820, Ocotlán, Jalisco, México. Teléfono: (+52-33)3686-2267, psolis@mexis.com 


\title{
Comparaçáo da saúde subjetiva entre protótipos de personalidade em populaçáo geral do México
}

\begin{abstract}
Resumo
O objetivo deste estudo foi obter protótipos da personalidade em população geral do México e comparar a saúde subjetiva entre eles. No total, participaram 994 indivíduos de 14 a 63 anos. Avaliaram-se cinco traços de personalidade (extroversão, neuroticismo, abertura à experiência, conscienciosidade e amabilidade) com o NEO-FFI, e a saúde subjetiva com o GHQ12, e uma pergunta sobre o estado de saúde. Para corroborar a consistência dos protótipos, dividiu-se a amostra em dois grupos de idade: jovem (de 14 a 25 anos) e adulto (de 26 a 63 anos). Como resultado, obtiveram-se três protótipos em ambos os grupos: as pessoas resilientes - baixo neuroticismo e alto nos demais traços-, os que tiveram a melhor saúde subjetiva; as não resilientes - alto neuroticismo e baixo no restante dos traços_-, que apresentaram a pior saúde subjetiva, e as pessoas disciplinadas —alto em conscienciosidade e média nos outros traços—, que apresentaram uma saúde subjetiva intermediária em comparação com os outros protótipos. Finalmente, a autodisciplina e a resiliência foram os traços que melhor discriminaram a boa saúde. Os achados são discutidos em termos da generalização de protótipos através de culturas.

Palavras-chave: Disciplinado, não resiliente, personalidade, protótipos, resiliente, saúde.
\end{abstract}

\section{INTRODUCCIÓN}

Una de las propuestas más representativas para el estudio de la personalidad y que ha logrado un considerable apoyo es la taxonomía de la personalidad representada por el modelo de los Cinco Grandes Factores de Personalidad -CGFP- (García, Aluja \& García, 2004; Hoyle, 2010). Probablemente, los instrumentos más reconocidos para operacionalizar el modelo de los CGFP son el Inventario NEO de la Personalidad - NEO PI-R - (Smith, Williams \& Segerstrom, 2015) y una versión breve del mismo conocida como NEO-FFI. Estos dos instrumentos, caracterizados por ser autorreportes, evalúan cinco dimensiones: Neuroticismo (N), que se caracteriza por la ansiedad, hostilidad, depresión, ansiedad social, impulsividad y vulnerabilidad; Extraversión (E), caracterizada por la cordialidad, gregarismo, asertividad, actividad, búsqueda de emociones y emoción positiva; Apertura a la experiencia $(\mathrm{O}$, por su sigla en inglés), relacionada con la fantasía, estética, sentimientos, acciones, ideas y valores; Amabilidad o Agradabilidad (A), relacionada con la confianza, franqueza, altruismo, actitud conciliatoria, modestia y sensibilidad a los demás; y, por último, Responsabilidad o Conciencia (C, por su sigla en inglés), entendida como el orden, sentido del deber, necesidad de logro y autodisciplina (Costa \& McCrae, 2008).

Los estudios con este modelo e instrumentación se pueden dividir en dos grandes enfoques (De Fruyt, Mervielde \& Van Leeuwen, 2002): el primero, que conceptualiza la personalidad en términos de las diferencias individuales entre las personas y que, por ejemplo, explora las relaciones de cada una de las dimensiones del NEO-FFI (N, E, O, A, y C) con diversos fenómenos psicológicos (Roberts, Kuncel, Shiner, Caspi \& Goldberg, 2007); y el segundo, de carácter tipológico o centrado en la persona, que se sustenta en el conjunto o configuración de atributos que definen a cada individuo y que, a partir de esta noción, puede agrupar a individuos con un patrón de personalidad semejante y explorar las relaciones con los fenómenos de interés (Xie, Chen, Lei, Xing \& Zhang, 2016).

Teniendo esto en cuenta, de acuerdo con Donellan y Robins (2010), los tipos de personalidad Resiliente, Sobrecontrolado y Subcontrolado fueron identificados por primera vez por Robins, John, Caspi, Moffitt y StouthamerLoeber (1996), y los estudios dicen que estos tres tipos son replicables, que tienen validez externa para predecir comportamiento problema, y que son útiles en la investigación y en la aplicación (Asendorpf, Borkenau, Ostendorpf \& Van Aken, 2001; De Fruyt et al., 2002; Van Leeuwen, De Fruyt \& Mervielde, 2004).

En cuanto a los rasgos sobresalientes de los tipos, las personas Resilientes muestran puntaje bajo en N, puntajes relativamente altos en el resto de las dimensiones, y se pueden describir como estables emocionalmente, asertivas y cordiales hacia otros. Por otra parte, los individuos Sobrecontrolados presentan alto puntaje en N y bajo en $\mathrm{E}$, con puntajes medios o relativamente bajos en las otras dimensiones, y se describen como emocionalmente inestables (ansiosos o tensos), e introvertidos. Mientras que quienes entran en la categoría de Subcontrolados presentan puntajes bajos en $\mathrm{A}$ y en $\mathrm{C}$, y puntajes medios en las otras dimensiones, además de que son individuos descritos con ausencia de control, búsqueda de emociones y orientados a las acciones (comportamiento antisocial). Con respecto a esto, múltiples hallazgos confirman que los tipos se asocian de manera diferencial con variables psicológicas y sociales diversas en todas las etapas del desarrollo de los individuos 
(Akse, Hale, Engels, Raaijmakers \& Meeus, 2007; Hart, Burock, London, Atkins \& Bonilla-Santiago, 2005; Klimstra, Hale, Raaijmakers, Branje \& Meeus, 2010; Steca, Alessandri \& Caprara, 2010; Van Leeuwen et al., 2004).

A pesar de lo anterior, la investigación con el enfoque centrado en la persona es incipiente, sobre todo en Iberoamérica $\mathrm{y}$, además, no es ajena a contradicciones. Con el presente trabajo no se pretende elaborar sobre todos los asuntos pendientes, ya que para ello existen excelentes revisiones (Caspi, 1998; Donellan \& Robins, 2010; Herzberg \& Roth, 2006; Miller, 2012), sino que lo que se busca es explorar las contradicciones sobresalientes y las posibles razones de las mismas. Así, es necesario considerar lo siguiente:

1. Algunos autores han señalado la falta de consistencia de los tipos. Por ejemplo, Herzberg y Roth (2006) muestran en su análisis de siete estudios que, a diferencia de las otras dimensiones, $\mathrm{N}$ es la única consistente en personas Resilientes y Sobrecontroladas. Estos autores transformaron los puntajes crudos en "calificaciones z" y encontraron que dimensiones como E y O del tipo Subcontrolado variaban enormemente en esas calificaciones (de $<.05 \mathrm{a}>.50$ ). El problema de no considerar estas variaciones se agrava cuando se etiqueta con los mismos nombres lo que pueden ser diferentes tipos, aparentando una homogeneidad de los mismos que dista mucho de ser perfecta (Herzberg \& Roth, 2006). Relacionado con lo anterior, Donellan y Robins (2010) reportan estudios que encuentran un prototipo "Indeseable", en lugar del Subcontrolado, que se asemeja al tipo inverso del Resiliente (alto puntaje en N y bajo en el resto de los rasgos). Pero parece que sería más adecuado nombrarlo No-Resiliente, como sugirieron Zawadzki y Strelau (2003). Igualmente, se han reportado configuraciones con cuatro y cinco tipos (Isler, Liu, Sibley \& Fletcher, 2016); por ejemplo, algunos de cinco tipos incluyen el tipo Seguro — puntajes relativamente altos en O y E- (Herzberg \& Roth, 2006) y, en otros casos, el Reservado o Discreto — con bajo puntaje en O- (Roth \& Von Collani, 2007), mientras que otros incluyen al Reservado y al Ordinario - puntajes medios en todo- (Kinnunen et al., 2012).

2. Un problema que puede relacionarse con el anterior es el uso del método de conglomerados, pues este ha sido el preferido particularmente con el análisis de Ward seguido de $K$-medias (Akse, Hale, Engels, Raaijmakers \& Meeus, 2004; Chapman \& Goldberg, 2011; Steca et al., 2010); pero Donellan y Robins (2010) argumentan que al usar este método no se realizan análisis de los datos múltiples, ni se obtienen submuestras. Por ejemplo, en los estudios revisados por Herzberg y Roth (2006), las muestras fluctuaban entre 156 y 786 individuos, pero en varios casos fueron divididas en mitades con el propósito de demostrar el criterio de replicabilidad de los tipos. Al hacer esto, las muestras fueron mucho menores a 500 y por ello resultan inadecuadas para obtener clasificaciones estables (Steinley, 2003). Adicionalmente, varias muestras no correspondían a la población general, ni eran homogéneas en alguna variable - como los universitarios, por ejemplo- y esto puede favorecer a que exista mayor o menor prevalencia de desordenes de personalidad y que, por tanto, se afecte la obtención de los tipos de personalidad (Donellan \& Robins, 2010).

3. También es de interés considerar que no todas las dimensiones que supuestamente caracterizan a cada tipo se comportan igual a través de estudios. Por ejemplo, para el tipo Resiliente, la mayoría de los estudios concuerdan en puntaje bajo en $\mathrm{N}$ y altos en el resto de los rasgos, pero otros trabajos indican puntajes promedio en todas las dimensiones y bajo en N (Van Leeuwen et al., 2004); o relativamente altos en E y C pero bajo en N (Ekehammar \&Akrami, 2003). También, para el Sobrecontrolado los estudios coinciden en puntaje alto en $\mathrm{N}$ y bajo en $\mathrm{E}$; pero algunos de estos indican, además, puntajes medios o bajos en el resto de los rasgos (Donellan \& Robins, 2010), o altos en N y C y bajos en E y O (Herzberg, 2009). Por otra parte, para el tipo Subcontrolado se encuentra una gran variabilidad y solo coinciden en puntaje bajo en $\mathrm{C}$ y, en algunos casos, en $\mathrm{A}$, con algunas otras excepciones (Donellan \& Robins, 2010; Ekehammar \& Akrami, 2003; Herzberg \& Roth, 2006). Por supuesto, estos hallazgos generan incertidumbre sobre las características de los tipos. También, un aspecto que suele omitirse en los estudios es el de "cuál es el criterio cuantitativo para identificar puntajes altos o bajos", y esto es importante porque, como sucede en un estudio en el que sí se especifican las desviaciones típicas por debajo o por encima de la media aritmética de los rasgos de la muestra para clasificar los tipos (De Fruyt et al., 2002), es posible y necesario evitar interpretaciones imprecisas sobre lo que significa "puntajes ligeramente bajos" o "puntajes relativamente altos".

4. Otros estudiosos han argumentado que los resultados contradictorios probablemente se deban a otras variables propias de las muestras, como el sexo, la edad, y la cultura (Herzberg \& Roth, 2006). Respecto al sexo, no se han encontrado evidencias consistentes de que esta variable se deba controlar, pero en cuanto a la edad, se conocen algunos estudios, como el de De Fruyt et al. (2002), donde no se recuperaron los tres tipos en las muestras de diferente edad, mientras que otros estudiosos argumentan que dado que los rasgos se relacionan con la edad, sus efectos son posibles en los prototipos (Strus, Cieciuch \& Rowinski, 2014). En cuanto a la cultura, en universitarios de Filipinas se recuperaron dos de los tres tipos más comunes tanto en 
mujeres como en hombres, pero se obtuvieron otros más, y los autores, Avdeyeva y Church (2005), concluyen que el apoyo para la generalización transcultural es parcial. Cabe mencionar que no existen estudios en Latinoamérica, pero en un estudio en España se replicaron estudios para identificar los tres tipos con una muestra estudiantil, aunque no con una de la población general (Boehm, Asendorpf \& Avia, 2002); y, recientemente, Alessandri et al. (2014) recuperaron los tres tipos en estudiantes de España, EUA, Italia y Polonia, con algunas variantes, en muestras de 235 a 499 universitarios, por lo que consideraron que la generalización transcultural era relativamente buena entre los países estudiados. De cualquier manera, es prematuro establecer conclusiones sobre el efecto de la cultura, ya que la mayoría de los estudios se ha realizado en países industrializados de occidente, particularmente en Europa. En contraste, en el primer estudio realizado en adolescentes de China, Xie et al. (2016) recuperaron solo el tipo Resiliente y el Subcontrolado (que tiene la menor consistencia en los estudios europeos), además del Ordinario y el Retraído.

5. Finalmente, se discute la importante distinción sobre la conceptualización de los tipos como etiquetas o como reflejo de clases que se encuentran en la naturaleza. La perspectiva generalizada ve a los tipos como etiquetas adecuadas para resumir la información de la personalidad, y se reconoce que no refleja categorías con sustrato biológico, sino más bien distinciones relativas entre las personas. Por estas y otras razones (Donellan \& Robins, 2010) parece más conveniente el uso del término "prototipo" que el de "tipo", como han sugerido varios autores (Weir \& Gjerde, 2002). El término prototipo indica preeminencia, sinónimo de primer molde o modelo, y no debe confundirse con un estatus definitivo o consolidado. Sin embargo, en la investigación se mencionan indistintamente como tipos, perfiles o prototipos de personalidad.

Teniendo en cuenta lo anterior, en el presente estudio se hizo la pregunta sobre si es posible recuperar los prototipos de personalidad en población general de México, y si se pueden corroborar diferencias en la salud subjetiva entre los prototipos recuperados. A pesar de que varios estudios apoyan la generalización transcultural del modelo de los CGFP, en la actualidad esta es cuestionada (Church, 2016), y dadas las consideraciones expuestas, replicar los estudios recuperando prototipos es de gran importancia en países de Latinoamérica porque, hasta donde se sabe, no se han aportado evidencias a favor o en contra de la generalización. En este contexto, parece prematuro establecer hipótesis; sin embargo, se esperaría recuperar por lo menos alguno de los tres prototipos más comunes (Resiliente, Sobrecontrolado y Subcontrolado) y, quizás, otros más.
Por otra parte, es de suma importancia comparar la salud subjetiva por prototipos porque los estudios en países desarrollados han comprobado que las personas con alto Neuroticismo perciben en sí mismos un estado de salud más pobre y reportan más enfermedades físicas que los individuos con menor puntaje en este rasgo. En general, el Neuroticismo se ha asociado con un impacto negativo en la salud tanto en medidas subjetivas como objetivas; mientras que para la Responsabilidad se ha encontrado lo contrario (Lahey, 2009; Roberts, Kuncel, Shiner, Caspi \& Goldberg, 2007).

Por ejemplo, Kinnunen et al. (2012) estudiaron la personalidad y la salud de 304 adultos e incluyeron medidas objetivas - masa corporal, presión, etc.- y subjetivas de la salud - una pregunta referente a cómo describirían los participantes su salud en el último año, una entrevista de salud para obtener síntomas, y el GHQ-12 como medida de distrés psicológico-. En dicho estudio se formaron prototipos con puntajes del NEO-FFI utilizando un análisis latente de perfiles (agrupaciones de rasgos), y los prototipos fueron: Resiliente - bajo $\mathrm{N}$ y alto en los otros rasgosSobrecontrolado - alto en N y bajo en E-, Subcontrolado - alto en $\mathrm{O}$ y E y bajo en $\mathrm{C}$ y N-, Reservado - bajo en $\mathrm{N}, \mathrm{E}, \mathrm{O}$ y A y alto en $\mathrm{C}-\mathrm{y}$ Ordinario - perfil cero en el modelo latente para todos los rasgos- Con respecto a las diferencias significativas, para las personas Resilientes se encontró el menor distrés psicológico (GHQ-12), el mejor puntaje de salud subjetiva y el menor número de síntomas; mientras que las personas Sobrecontroladas mostraron mayor distrés, peor puntaje de salud subjetiva y mayor número de síntomas. Los otros tres perfiles quedaron entre estos dos extremos.

En suma, el objetivo del presente estudio fue recuperar los prototipos de personalidad en población general de México y comparar la salud subjetiva entre ellos. Se planeó incluir, además del GHQ-12 como medida de la salud o distrés psicológico, una pregunta semejante a la hecha por Kinnunen et al. (2012). Cabe agregar que se consideraron análisis por sexo debido a las evidencias que indican que los hombres presentan una mejor salud percibida que las mujeres, pues estas presentan síntomas psicológicos y físicos con mayor frecuencia (Kaleta, Polańska, DziankowskaZaborszcsyk, Hanke \& Drygas, 2009); sin embargo, no se consideraron análisis por sexo para los prototipos porque en los autorreportes con diferentes versiones del instrumento de personalidad aquí utilizado no se reportan diferencias por sexo consistentes (Borkenau, Hrebícková, Kuppens, Realo $\&$ Allik, 2013). Se espera que los resultados ofrezcan, por primera vez en Latinoamérica, conocimiento sobre el tema. 


\section{MÉTODO}

El presente consiste en un estudio con diseño transversal descriptivo.

\section{Participantes}

Teniendo en cuenta que se revisó el estado de arte de los estudios sobre prototipos, para recuperarlos se planeó obtener una muestra mayor y dividirla por edad, y así tener dos grupos: uno joven y otro maduro. En total, se calculó una muestra de 1440 participantes mediante muestreo no probabilístico por conglomerados, utilizando como criterios el sexo y la edad cronológica. De estos, la tasa de respuesta fue del $69 \%$, por lo que la muestra definitiva quedó compuesta por 994 sujetos, quienes radicaban en 13 municipios de cuatro diferentes Estados de México: Jalisco, Veracruz, Colima y Michoacán.

Las edades abarcaban de los 14 a los 63 años, los varones $(n=413)$ tenían una edad media de 31.6 años $(D E=$ $13.1)$ y las mujeres $(n=581)$ de 28.3 años $(D E=11.8)$; los porcentajes de las principales variables sociodemográficas fueron:

- Estado civil: $64.6 \%$ solteros, $29.0 \%$ casados, $3.7 \%$ con pareja, $1.7 \%$ divorciados y $1.0 \%$ viudos.

- Nivel educativo: $1.1 \%$ sin estudios, $5.1 \%$ elemental, $9.2 \%$ secundario, $25.3 \%$ bachillerato, $14.4 \%$ técnico, $38.6 \%$ licenciatura y $6.3 \%$ posgrado.

- Actividad que realiza actualmente: $51.7 \%$ estudiante, $9.4 \%$ obrero, $10.2 \%$ hogar, $9.6 \%$ comerciante, $3.4 \%$ técnico, y $15.8 \%$ profesionista.

Ningún participante reportó antecedentes de afectaciones mentales o psiquiátricas, y todos firmaron la respectiva carta de consentimiento informado. Para los participantes menores de edad $(7.5 \%)$ sus padres firmaron consentimiento informado.

\section{Instrumentos}

Cuestionario socio demográfico. Se preguntó por la edad, el estado civil, la escolaridad y la ocupación actual. Además, se pidió que se contestaran a la pregunta "¿padece algún problema de salud?".

Cuestionario General de Salud (GHQ-12). Para valorar la salud subjetiva se eligió la versión de 12 ítems del General Health Questionnaire (GHQ-12) de Goldberg y Williams (1988). Este instrumento permite evaluar la salud auto percibida y el estado de bienestar general (Goldberg \& Williams, 1988) y es reconocido por su utilidad como una medida de tamizaje del estado subjetivo de salud general y de algunas disfunciones mentales. Particularmente, se presenta en escala Likert con cuatro opciones de respuesta: nunca (0), a veces (1), casi siempre (2) y siempre (3). Y, en este estudio, los 12 ítems se dividieron con base en su redacción en formato positivo o negativo, quedando con seis ítems cada subescala; en donde a mayor puntaje, mayor disfunción. Para fines descriptivos, la confiabilidad alfa de Cronbach total fue de .80; para los ítems positivos, de .85; y para los negativos, de .82. Otros autores han reportado valores alfa de $.81, .85$, y .82 , respectivamente (Solís-Cámara et al., 2016). La versión utilizada fue la misma con la que se demostró la estructura factorial del GHQ-12 en población general de México (Solís-Cámara et al., 2016).

Cabe mencionar que el GHQ es probablemente el instrumento más reconocido a nivel mundial para valorar el grado de salud percibida, y la versión de 12 ítems se ha identificado como la de mayor utilización por su facilidad de administración (Sánchez-López \& Dresch, 2008); además de que es un instrumento de tamizaje de la salud mental que se asocia significativamente con múltiples desórdenes físicos (González et al., 2012; Tuuliainen/Kirsi Sipilä, Mäki, Könönen \& Suominen, 2015), y ha sido la versión recomendada como la más adecuada para encuestas de salud (McDowell, 2006). Sin embargo, parece necesario tomar en cuenta que Hankins (2008) demostró que los análisis factoriales que apoyan la naturaleza multidimensional del GHQ-12 no toman en cuenta que los ítems redactados en formato negativo generan divisiones espurias de factores (dos o tres), debidas a efectos de las respuestas al formato negativo. Los estudios en muchos países, incluyendo México, han confirmado este error del método en el GHQ12, y han sugerido, entre otras opciones, separar los ítems positivos y negativos para contar con un método general de tamizaje de la salud subjetiva o el malestar psicológico (Smith, Oluboyede, West, Hewison \& House, 2013; SolísCámara, Meda Lara, Moreno-Jiménez \& Juárez, 2016; Urzúa, Caqueo-Urizar, Bargsted \& Irarrázaval, 2015).

Inventario NEO Reducido de Cinco Factores (NEO-FFI) de Costa y McCrae (2008). Se utilizó la versión española (Costa \& McCrae, 2008) porque en la fecha de realización del estudio no existía ninguna versión validada en México, sin embargo, recientemente se propuso un versión de 30 ítems (Meda-Lara, Moreno-Jiménez, García, PalomeraChávez \& Mariscal de Santiago, 2015). El NEO-FFI evalúa las dimensiones del modelo CGFP: Neuroticismo, Extraversión, Apertura a la experiencia, Amabilidad y Responsabilidad, conocidas por sus siglas en inglés como $\mathrm{N}, \mathrm{E}, \mathrm{O}, \mathrm{A}$ y $\mathrm{C}$, respectivamente. La cual es aplicable a adultos y adolescentes, y consta de 60 ítems divididos en las cinco escalas, cada una con 12 ítems. 
El NEO-FFI se presenta en formato tipo Likert de cinco puntos: totalmente de acuerdo, de acuerdo, neutral, en desacuerdo y total desacuerdo; en donde, tras la calificación, puntuaciones altas indican mayor expresión del rasgo. Los valores de fiabilidad alfa obtenidos en este estudio fueron: $\mathrm{N}(.78), \mathrm{E}(.81), \mathrm{O}(.73), \mathrm{A}(.71), \mathrm{C}(.81)$ y si se considera el valor 75 como fiabilidad aceptable, tres dimensiones presentaron fiabilidad aceptable y dos regular o media (Hernández, Fernández \& Baptista, 2010). Otros estudios reportan valores de $.86, .77, .73, .68$ y .81 (Costa \& McCrae, 2008); o .83, .78, $.67, .67$ y .84 , respectivamente (Martínez \& Cassaretto, 2011).

Cabe mencionar que se eligieron el NEO-FFI, para valorar la personalidad, y el método de análisis de conglomerados para recuperar los prototipos; además, se siguió el criterio cuantitativo de De Fruyt et al. (2002) para identificar los puntajes de los prototipos. En general, el Resiliente se identificó por puntaje bajo en $\mathrm{N}$ (media desviación estándar por debajo del promedio) y puntajes altos $(+1 / 2 D E)$ en las otras dimensiones; el Sobrecontrolado por un puntaje alto en $\mathrm{N}(+1 / 2 D E)$, bajo en $\mathrm{E}(-1 / 2 D E)$ y promedio en el resto de las dimensiones; y el Subcontrolado por un puntaje bajo en $\mathrm{C}$ (entre $-1 / 2$ y $1 D E$ ) y en $\mathrm{A}(-1 / 2 D E)$ y promedio en las otras dimensiones.

\section{Procedimiento}

Los instrumentos y la carta de consentimiento informado fueron convertidos a formato electrónico con el software SurveyMonkey; los cuales fueron enviados a la dirección de correo electrónico de potenciales participantes que fueron principalmente estudiantes y profesores universitarios, amas de casa, obreros y comerciantes que residían en los Estados de México reportados anteriormente. Es importante mencionar que en el presente trabajo se tomaron en cuenta la Declaración de Helsinki de 2004 y el Código Ético del Psicólogo (Sociedad Mexicana de Psicología, 2007), cuidando la integridad de los participantes y el uso confidencial de los datos. El proyecto fue avalado por el Consejo de Ética de la Universidad de Guadalajara.

\section{Análisis de datos}

Los análisis se realizaron con el paquete estadístico SPSS-21. Se obtuvieron las fiabilidades de las subescalas del NEO-FFI y por separado para los ítems positivos y negativos del GHQ-12 con el coeficiente alfa de Cronbach. Adicionalmente, se analizaron la asimetría y la curtosis de las principales variables del estudio para conocer su distribución de acuerdo a la curva normal.

Como se mencionó anteriormente, la muestra fue dividida en dos grupos de edad (joven y maduro) con el propósito de replicar los prototipos extraídos. Para la identificación de los prototipos con el método de conglomerados se siguieron varios procedimientos, semejantes para ambos grupos: el modelo con algoritmo bietápico con las variables continuas $\mathrm{N}, \mathrm{E}, \mathrm{O}, \mathrm{A}$ y $\mathrm{C}$, variables asumidas como tipificadas, y medida de distancias euclidianas con número de agrupaciones automática (hasta 15) y con manejo del ruido (25\%).

Primero, se consideró el resumen del conglomerado bietápico, que incluyó en ambos grupos las cinco dimensiones; luego, se tomó en cuenta la medida de silueta de cohesión y comparación con el criterio de calidad del conglomerado entre $>.5$ y lo más cercano a 1.0, es decir, calidad buena, para elegir las soluciones de esos análisis a incluir en el Método de Ward. A continuación, se realizó un análisis con el Método de Ward y posteriormente el de K-medias sin actualización de medias. Los prototipos recuperados fueron analizados vía $C h i^{2}$ para determinar diferencias en el tamaño de los mismos y la distribución de los sexos. Para determinar efectos por edad, sexo y su interacción se incluyó un Anova de un factor y análisis univariados; mientras que para los análisis post-hoc se eligió un método conservador (Scheffe) y uno adecuado para corregir la significación por múltiples comparaciones (GT2 de Hochberg).

Finalmente, para conocer las diferencias por prototipos en la salud subjetiva, los puntajes fueron convertidos a "calificaciones z" con el propósito de homogeneizar las variables, tal como se aplicó en otros estudios (Kinnunen et al., 2012). Y, con respecto a las diferencias en los puntajes del GHQ-12 y por sexo, se utilizó la prueba $t$ de Student para muestras independientes, incluyendo la prueba Levene de igualdad de varianza, el error típico y los intervalos de confianza.

\section{RESULTADOS}

A continuación se presentan los análisis descriptivos de la muestra divididos por grupo de edad; posteriormente, los análisis de conglomerados que permitieron obtener tres prototipos de personalidad, los cuales se analizan en detalle en cuanto a las cinco dimensiones de personalidad; $y$, finalmente, se presentan las comparaciones en salud subjetiva por prototipos y por sexo en los dos grupos de edad.

La muestra se dividió en dos grupos de edad: joven, (GJ) de 14 a 25 años $(M=19.9, D E=2.43)$, y maduro $(\mathrm{GM})$, de 26 a 63 años $(M=41.2, D E=9.42)$. El primero $(n=541)$ quedó conformado por 340 mujeres $(62.8 \%)$ y 201 hombres $(37.2 \%)$, y el segundo $(n=453)$ por 241 mujeres $(53.2 \%)$ y 212 hombres $(46.8 \%)$. En la Tabla 1 se muestran, con fines descriptivos, las medias, asimetrías y curtosis de las variables de interés para el total de la muestra y por grupo de edad. Adicionalmente, se observa que las asimetrías y curtosis de la mayoría de las variables 
se ajustan adecuadamente a la curva normal, e indican que la edad se concentraba ligeramente en una muestra joven y que las respuestas a la pregunta "¿padece algún problema de salud?" se concentraban en la respuesta "no" (71\%), particularmente en el GJ, que presenta una media de 1.80 . Por otra parte, se observa una ligera asimetría positiva para GHQ-ítems negativos, lo que indica que los valores tendieron a agruparse en puntajes bajos.

\section{Formación de los prototipos}

El procedimiento fue semejante para ambos grupos. En el grupo joven los resúmenes de los análisis de conglomerado bietápico indicaron dos soluciones, donde la medida de silueta de cohesión y comparación indicó que la calidad del conglomerado era $>.05$ y cercana a 1.0 , es decir, buena. También, se obtuvieron soluciones de dos y tres conglomerados con el Método de Ward, donde la solución con dos prototipos incluía uno con puntaje alto en $\mathrm{N}$ y puntajes bajos en los otros rasgos, mientras que para el otro era el opuesto. Finalmente, se retuvo la solución de tres conglomerados para análisis posteriores porque la solución de dos pareció ser menos enriquecedora.

Por otra parte, en el grupo maduro los resúmenes de los análisis de conglomerado bietápico indicaron cuatro soluciones con dos, tres, cuatro y cinco conglomerados, y la medida de silueta de cohesión y comparación indicó que la calidad de cada conglomerado era $>.05$. De manera similar, las soluciones con tres, cuatro y cinco conglomerados fueron más cercanas a 1.0. Posteriormente, análisis con el método de Ward permitieron observar que las soluciones de cuatro y cinco conglomerados eran semejantes en tres prototipos, pero los otros dos presentaban altos o bajos puntajes en todos los rasgos. Finalmente, se conservaron tres conglomerados, ya que esta opción ofrecía una solución más consistente, por lo menos con dos de los tres conglomerados comunes, además de que era semejante en ambos grupos de edad.

\section{Descripción de los prototipos}

En la Tabla 2 se presentan los puntajes del GJ en el NEO-FFI por prototipos. En resumen, el prototipo Resiliente se caracterizó por un puntaje con una desviación estándar por debajo de la media en Neuroticismo, y puntajes altos en Extraversión $(+1 D E)$, Apertura $(+1 / 2 D E)$, Amabilidad $(+1 / 2 D E)$ y Responsabilidad $(+3 / 4 D E)$. El segundo prototipo se caracterizó por presentar un puntaje con media desviación por encima del promedio en Neuroticismo y puntajes bajos en Extraversión (-1/2 DE), Apertura (-1/2 $D E)$, Amabilidad (-1/2 $D E)$ y Responsabilidad (-3/4 $D E)$, y por ello fue nombrado No-Resiliente. El tercer prototipo presentó puntajes promedio (ni siquiera media $D E$ por debajo o encima de la media) en todas las dimensiones, con excepción del puntaje alto en Responsabilidad (casi $+3 / 4$ $D E)$; este prototipo fue nombrado Disciplinado porque dicha dimensión refiere a rasgos de orden, organización y autodisciplina.

El tamaño de los prototipos fue diferente $\left(X^{2}(2)=56.0\right.$, $p<.000$ ), con $28.3 \%$ Resilientes, $48.2 \%$ No-Resilientes y $23.5 \%$ Disciplinados. Y se encontró que las mujeres estaban sobre representadas, pues en el prototipo Resiliente había 99 mujeres y 54 hombres; en el No-Resiliente había 148 mujeres y 113 hombres; y en el Disciplinado, 93 mujeres y 34 hombres. Finalmente, no se observaron diferencias de edad entre los prototipos $\left(F_{(2,538)}=.88, p>.05\right)$, ni interacción significativa de la edad por prototipo y sexo $\left(F_{(2,541)}=.81, p>.05\right)$.

En la Tabla 3 se presentan los puntajes del GM por prototipos. En general, el prototipo Resiliente se caracterizó por

Tabla1

Estadisticas descriptivas y asimetría y curtosis de las variables para la muestra total y sus medias por grupo

\begin{tabular}{|c|c|c|c|c|c|c|c|c|}
\hline \multirow[b]{2}{*}{ Variables } & \multicolumn{4}{|c|}{ Total } & \multicolumn{2}{|c|}{ GJ } & \multicolumn{2}{|c|}{ GM } \\
\hline & $M$ & $D E$ & A & $\mathrm{C}$ & $M$ & $D E$ & $M$ & $D E$ \\
\hline Edad en años. & 29.7 & 12.5 & .80 & -.69 & 28.3 & 11.8 & 31.5 & 13.1 \\
\hline Problema de salud. & 1.71 & .45 & -.93 & -1.14 & 1.80 & .39 & 1.60 & .49 \\
\hline GHQ-ítems positivos. & 7.28 & 3.79 & -.02 & -.23 & 6.85 & 3.82 & 7.78 & 3.70 \\
\hline GHQ-ítems negativos. & 5.58 & 3.94 & .81 & .19 & 6.05 & 4.05 & 5.00 & 3.71 \\
\hline Neuroticismo. & 20.8 & 7.45 & .12 & -.04 & 21.4 & 7.59 & 20.2 & 7.23 \\
\hline Extraversión. & 29.8 & 7.49 & -.28 & .21 & 30.0 & 7.89 & 29.6 & 6.99 \\
\hline Apertura. & 27.6 & 6.58 & .31 & .07 & 28.3 & 6.42 & 26.7 & 6.68 \\
\hline Amabilidad. & 28.1 & 6.50 & -.12 & -.02 & 27.7 & 6.56 & 28.5 & 6.41 \\
\hline Responsabilidad. & 30.2 & 7.08 & -.04 & -.23 & 30.2 & 7.37 & 30.3 & 6.73 \\
\hline
\end{tabular}

Nota. Total $(n=994)$. GJ = grupo joven. $\mathrm{GM}=$ grupo maduro. $\mathrm{A}=$ asimetría. $\mathrm{C}=$ curtosis. $\mathrm{GHQ}=$ Cuestionario General de Salud. 
presentar el puntaje más bajo de todos en Neuroticismo (-1 $D E)$, y puntajes altos en el resto de las dimensiones, todos ellos aproximadamente con una desviación por encima de la media. El No-Resiliente se caracterizó por presentar el puntaje más alto en Neuroticismo $(+1 / 2 D E)$, y puntajes bajos en Extraversión (-3/4 $D E)$, Apertura (-1/2 $D E)$, Amabilidad (-1/2 DE), y Responsabilidad (-3/4 DE). El Disciplinado se caracterizó por presentar puntajes cercanos a la media o ligeramente por encima de la misma $(<$ de $1 / 2 D E)$ en todas las dimensiones, con excepción del puntaje alto en Responsabilidad $(+1 / 2 D E)$.

El tamaño de los prototipos fue claramente diferente, con $18.1 \%$ Resilientes, $44.8 \%$ No-Resilientes y $37.1 \%$ Disciplinados. Y en el Resiliente $\left(X^{2}(1)=3.12, p=.07\right)$ habían menos mujeres (33) que hombres (49); en el de No-Resilientes las mujeres estaban sobrerrepresentadas (118) de manera significativa $\left(X^{2}(1)=5.36, p<.05\right)$, en comparación con los hombres (85); y en el Disciplinado, el sexo (90 mujeres y 78 hombres) se distribuyó de manera más homogénea $\left(X^{2}(1)=.86, p=.35\right)$. Adicionalmente, se observaron diferencias de edad entre los prototipos $\left(F_{(2,453)}=3.75, p<.05\right)$ y por sexo $\left(F_{(1,453)}=10.0, p<.01\right)$, pero no interacción significativa por prototipo y sexo $\left(F_{(2,453)}=2.63, p>.05\right)$. Finalmente, los análisis post-hoc con los métodos Scheffe y de Hochberg fueron significativos $(p<.05)$, e indicaron que el prototipo Disciplinado tenía menor edad $(M=39.6, D E=8.67)$ que el No-Resiliente
( $M=42.4, D E=9.6)$; y que las mujeres $(M=39.5)$ tenían menor edad que los hombres $(M=42.6)$.

\section{Diferencias en salud por prototipos}

Ante la pregunta sobre el estado de salud y los ítems positivos y negativos del GHQ-12, las respuestas se convirtieron en valores z con el propósito de homogeneizar las variables de salud subjetiva y compararlas por prototipos y por sexo en el GJ y el GM. Todas las diferencias de análisis univariados incluyeron una prueba post-hoc conservadora (Scheffe) y la prueba Levene sobre igualdad de varianzas, las cuales se mencionan solo en los casos significativos (véase Tabla 4). Es importante aclarar que las comparaciones se mencionan como puntajes menores o mayores con el propósito de facilitar la comunicación de los resultados, pero se reconoce que se trata de un número de unidades por debajo (negativos) o por encima de la media (positivos). La primera comparación significativa se presenta con ejemplos para aclarar más las subsecuentes.

Con respecto a la pregunta sobre el estado de salud, en el GJ no se encontraron diferencias significativas. Sin embargo, las diferencias en GHQ-positivos y GHQ-negativos por prototipos fueron semejantes, pero más claras para los positivos que para los negativos, por esto, solo se presentan dichos análisis. Específicamente, el Resiliente tuvo el menor puntaje en GHQ-positivos (véase Tabla 4); es decir, el mayor número de unidades o desviaciones (-.72) por debajo de

Tabla 2

Puntajes de las dimensiones de personalidad por prototipos en el grupo joven

\begin{tabular}{ccccccc}
\hline & \multicolumn{2}{c}{ Resiliente } & \multicolumn{2}{c}{ No-Resiliente } & \multicolumn{2}{c}{ Disciplinado } \\
Variables & $M$ & $D E$ & $M$ & $D E$ & \multicolumn{2}{c}{ DE } \\
\hline Neuroticismo (N) & 14.3 & 5.04 & 26.1 & 6.13 & 20.5 & 5.44 \\
Extraversión (E) & 38.2 & 4.11 & 25.9 & 7.02 & 28.6 & 5.17 \\
Apertura (O) & 32.1 & 5.68 & 26.3 & 5.78 & 27.7 & 6.54 \\
Amabilidad (A) & 31.9 & 6.61 & 24.2 & 5.40 & 29.9 & 4.49 \\
Responsabilidad (C) & 35.1 & 5.73 & 25.2 & 5.97 & 34.5 & 4.43 \\
\hline
\end{tabular}

Nota. Resiliente $(n=153)$, No-Resiliente $(n=261)$, Disciplinado $(n=127)$.

Tabla 3

Puntajes de las dimensiones de personalidad por prototipos en el grupo maduro

\begin{tabular}{ccccccc}
\hline & \multicolumn{2}{c}{ Resiliente } & \multicolumn{2}{c}{ No-Resiliente } & \multicolumn{2}{c}{ Disciplinado } \\
Variables & $M$ & $D E$ & $M$ & $D E$ & $M$ & $D E$ \\
\hline Neuroticismo (N) & 11.7 & 4.08 & 24.4 & 5.71 & 19.1 & 5.99 \\
Extraversión (E) & 37.0 & 4.74 & 24.3 & 5.08 & 32.3 & 4.76 \\
Apertura (O) & 34.8 & 5.50 & 23.4 & 5.10 & 26.8 & 5.43 \\
Amabilidad (A) & 34.2 & 4.86 & 25.1 & 5.22 & 29.9 & 5.89 \\
Responsabilidad (C) & 36.9 & 5.33 & 25.2 & 4.25 & 33.1 & 5.13 \\
\hline
\end{tabular}

Nota. Resiliente $(n=82)$, No-Resiliente $(n=203)$, Disciplinado $(n=168)$. 
la media, en comparación con los prototipos Disciplinado y No-Resiliente; lo que indica mejor salud general o menor malestar percibido. Además, cabe mencionar que el Disciplinado tuvo también mayor número de unidades (-.19) por debajo de la media que el No-Resiliente (.29).

Por otro lado, la diferencia por sexo (véase Tabla 4) se debió a que los hombres $(n=201)$ tuvieron menor puntaje en el GHQ-positivos $(M=-.436$, Error típico $=.072$; IC $95 \%=-.576 /-.295)$ que las mujeres $(n=340 ; M=-.112$, Error típico $=.050$; IC $95 \%=-.210 /-014)$; es decir, que estas percibieron más problemas de salud. También, se realizaron pruebas t para muestras independientes con el fin de identificar las interacciones prototipo-sexo en el GHQ-positivos; acá, el puntaje de los hombres $(M=-.716$, $D E=.87)$ fue menor que el de las mujeres $(M=-.000$, $D E=.86)$ únicamente en el prototipo Disciplinado $\left(t_{(125)}=-4.11, p<.001\right.$; diferencia de medias $=-.716$; IC $95 \%$ para la diferencia $=-1.06 /-.371)$, lo que apunta a que las mujeres percibieron más problemas de salud en este prototipo.

Debido a la influencia del sexo en las comparaciones anteriores se pensó en la conveniencia de explorar más dicha influencia y, por ello, se realizaron comparaciones intrasexo. Para las mujeres, se encontraron diferencias entre las Resilientes $(M=-.672, D E=.82)$ y las No-Resilientes $(M=$ $.335, D E=.97 ;\left(t_{(245)}=-8.420, p<.001\right)$ y las Disciplinadas $\left(M=-.000, D E=.86 ; t_{(190)}=-5.488, p<.001\right) ; y$ se encontró una diferencia entre estos dos últimos prototipos $\left(t_{(239)}=\right.$ $2.702, p<.01)$, aunque en todas estas comparaciones el $N o$ Resiliente mostró peor salud subjetiva y el Resiliente tuvo significativamente menor puntaje que los otros prototipos $y$, por lo tanto, mejor salud subjetiva. Por otro lado, en el caso de los hombres, los Resilientes $(M=-.815, D E=.79)$ y los Disciplinados $(M=-.716, D E=.87)$ tenían puntajes semejantes $\left(t_{(86)}=.543, p=.58\right)$; $\mathrm{y}$ tanto los Resilientes $\left(t_{(165)}\right.$ $=7.04, p<.001)$ como los Disciplinados $\left(t_{(145)}=5.23, p<\right.$ $.001)$ tenían puntajes menores que los No-Resilientes $(M=$ $.224, D E=.93)$; de esto se concluye que, en los hombres, los No-Resilientes también mostraron peor salud subjetiva.

Adicionalmente, para el GM no se encontró efecto o interacción por sexo en las comparaciones, aunque se encontraron diferencias significativas por prototipos para la pregunta "¿presenta actualmente algún problema de salud?”. Dado que la pregunta se contestó con un "sí" o un "no", las respuestas positivas indicaron un valor $\mathrm{z}$ negativo más grande (véase Tabla 4). Además, la prueba Levene sobre igualdad de las varianza fue significativa $\left(F_{(5,447)}=8.148\right.$, $p<.001)$, y los individuos Resilientes $(M=-.122$, Error típico $=.121 ;$ IC $95 \%=-.360 / .116)$ obtuvieron un puntaje semejante a los Disciplinados $(M=-.113$, Error típico = .083 ; IC $95 \%=-.276 / .050)$, aunque solo los Resilientes tenían puntaje significativamente menor $(p<.05)$ que los No-Resilientes $(M=-.390$, Error típico $=.076$; IC $95 \%$ $=-.540 /-.240$ ) tanto con la prueba Scheffe como en la de

Tabla 4

Diferencias en salud subjetiva por prototipos y por sexo en los grupos joven y maduro

\begin{tabular}{|c|c|c|c|c|c|c|c|c|c|}
\hline \multirow[b]{2}{*}{ Grupo Joven } & \multicolumn{2}{|c|}{$\begin{array}{l}\text { 1. Resiliente } \\
(n=153)\end{array}$} & \multicolumn{2}{|c|}{$\begin{array}{l}\text { 2. No-Resiliente } \\
(n=261)\end{array}$} & \multicolumn{2}{|c|}{$\begin{array}{l}\text { 3. Disciplinado } \\
(n=127)\end{array}$} & \multirow{2}{*}{$\begin{array}{l}\text { Diferencias prototipos } \\
\qquad F(2,541)\end{array}$} & \multirow{2}{*}{$\begin{array}{l}\text { Efecto del sexo } \\
\qquad F(1,541)\end{array}$} & \multirow{2}{*}{$\begin{array}{l}\text { Interacción } \\
F(2,541)\end{array}$} \\
\hline & $M$ & $D E$ & $M$ & $D E$ & $M$ & $D E$ & & & \\
\hline Problema de salud & .31 & .80 & .14 & .92 & .26 & .85 & 1.92 & 1.06 & .017 \\
\hline GHQ-positivos & -.72 & .81 & .29 & .95 & -.19 & .92 & $62.1 * * * 1<2,3 ; 3<2$ & $13.7 * * *$ & $4.34 * *$ \\
\hline \multirow[t]{2}{*}{ GHQ-negativos } & -.43 & .72 & .54 & 1.05 & -.06 & .92 & $52.3 * * * 1<2,3 ; 3<2$ & $12.3 * * *$ & $3.27 * *$ \\
\hline & \multicolumn{2}{|c|}{$\begin{array}{l}\text { 1. Resiliente } \\
(n=82)\end{array}$} & \multicolumn{2}{|c|}{$\begin{array}{l}\text { 2. No-Resiliente } \\
\qquad(n=203)\end{array}$} & \multicolumn{2}{|c|}{$\begin{array}{l}\text { 3. Disciplinado } \\
(n=168)\end{array}$} & Diferencias prototipos & Efecto del sexo & Interacción \\
\hline Grupo Maduro & $M$ & $D E$ & $M$ & $D E$ & $M$ & $D E$ & $F(2,453)$ & $F(1,453)$ & $F(2,453)$ \\
\hline Problema de salud & -.11 & 1.05 & -.39 & 1.10 & -.12 & 1.05 & $3.56^{*} 1<2$ & 1.29 & .51 \\
\hline GHQ-positivos & -.56 & .84 & .57 & .88 & -.06 & .88 & $52.0 * * * 1<2,3 ; 3<2$ & 3.14 & .63 \\
\hline GHQ-negativos & -.63 & .67 & .12 & .99 & -.23 & .88 & $19.6^{* * *} 1<2,3 ; 3<2$ & 1.57 & .20 \\
\hline
\end{tabular}

Nota. Para "problema de salud" a mayor puntaje negativo, los grupos aceptaron presentar problemas de salud. Para GHQ a mayor puntaje positivo, los grupos presentaron mayor problema de salud. ${ }^{* * *} .001 ; * * .01 ; * .05$. 
Hochberg. Aunque esta diferencia parece marginal, los porcentajes de respuestas "sí" a la pregunta de salud, según los prototipos, fueron claramente diferentes: Resiliente (15.5), No-Resiliente (46.8) y Disciplinado (32.0).

Las diferencias por prototipos, donde el Resiliente tuvo el menor puntaje en GHQ-positivos (véase Tabla 4), es decir, el mayor número de unidades o desviaciones (-.56) por debajo de la media comparados con el Disciplinado y el No-Resiliente, indican mejor salud general o, si se prefiere, menor malestar percibido. Además, el prototipo Disciplinado tuvo también mayor número de unidades (-.06) por debajo de la media que el No-Resiliente, cuyas unidades estaban por encima de la media (.57).

\section{DISCUSIÓN}

El objetivo del presente estudio fue recuperar los prototipos de personalidad en población general de México y comparar la salud subjetiva entre ellos. En este campo, el método más común para extraer prototipos ha sido el análisis de conglomerados; considerando esta estrategia como un tipo de replicación o validación de los prototipos recuperados, para aplicarla se tuvo en cuenta que este método es dependiente de las características muestrales, por lo que suelen utilizarse los criterios de hacer uso de una gran muestra dividida en dos o la inclusión de dos muestras para confirmar los prototipos (Herzberg \& Roth, 2006). Además, aunque los efectos de la edad sobre los prototipos no son consistentes, para minimizar sus posibles efectos en la composición de la muestra, dicha composición fue dividida arbitrariamente en dos grandes grupos de edad: el grupo joven (GJ), con edades en un rango estrecho (14-25 años), y el grupo maduro (GM), con un rango de edades muy amplio (26-63 años).

En general, se obtuvieron tres prototipos consistentes en ambos grupos. Sin embargo, no se encontró el prototipo Subcontrolado, que como se anotó anteriormente, es el menos consistente. Particularmente, dos de los prototipos recuperados correspondieron a los comúnmente extraídos en un gran número de países, particularmente en Europa (Alessandri et al., 2014; Donellan \& Robins, 2010; Herzberg \& Roth, 2006): en ambos grupos de edad, el Resiliente se caracterizó por un puntaje bajo en Neuroticismo (N) y puntajes altos en las dimensiones de Apertura a la experiencia $(\mathrm{O})$, Amabilidad (A) y, sobre todo, en Extraversión (E) y Responsabilidad (C); configuración semejante a la de otros estudios (Herzberg, 2009; Herzberg \& Roth, 2006; Kinnunen et al., 2012).
El prototipo tradicionalmente conocido como Sobrecontrolado ha sido nombrado en otros estudios como Indeseable —o No-deseable, si se prefiere evitar la denominación peyorativa (Barbaranelli, 2002)—, sin embargo, en este trabajo se nombró como No-Resiliente, tal como lo propusieron Zawadzki y Strelau (2003), ya que es claramente el opuesto del Resiliente (Alessandri et al., 2014) y porque no presenta alto puntaje en Responsabilidad, el cual se ha relacionado con el autocontrol (Strus et al., 2014), y que justificaría nombrarlo Sobrecontrolado. El presente trabajo se caracterizó por presentar el puntaje más alto en Neuroticismo y los puntajes más bajos en el resto de los rasgos, particularmente en Responsabilidad; configuración semejante a la encontrada en otras investigaciones (Ekehammar \& Akrami, 2003).

El tercer prototipo extraído presentó puntajes cercanos al promedio, o ligeramente altos (que no alcanzan $1 / 2 D E$ por encima de la media) en todas las dimensiones, con excepción de la Responsabilidad, donde el puntaje fue más alto, y de ahí que fuera nombrado como prototipo Disciplinado; los puntajes de todas las dimensiones de este grupo fueron intermedios en comparación a los de los otros dos prototipos.

Aunque pareciera, a primera vista, que este prototipo puede confundirse con "el auténtico" Sobrecontrolado, por su puntaje alto en Responsabilidad (entre $+1 / 2$ y $+3 / 4 D E$ ) este no cumple con el bajo puntaje en Extraversión que la mayoría de los estudios apoyan (Donellan \& Robins, 2010; Herzberg \& Roth, 2006). De hecho, muchos estudios que indican haber recuperado el prototipo Sobrecontrolado no muestran acuerdo en el puntaje de Responsabilidad, pues en algunos fue bajo (Barbaranelli, 2002; De Fruyt et al., 2002), en otros promedio (Asendorpf et al., 2001; Boehm et al., 2002), y en otros alto (Van Leeuwen et al., 2004). Indudablemente una de las razones de los hallazgos contradictorios mencionadas ampliamente en este trabajo.

Por otra parte, en el grupo joven los porcentajes de personas Resilientes y Disciplinadas fueron semejantes, pero en No-Resilientes el porcentaje fue el doble. Muy diferente fue la distribución del grupo maduro, ya que un porcentaje mínimo correspondió a individuos Resilientes, pero los No-Resilientes concentraron a la mayoría de las personas con un porcentaje intermedio de individuos Disciplinados. $\mathrm{Y}$ aunque en algunos estudios los prototipos se distribuyen homogéneamente, una tercera parte de cada uno (Herzberg, 2009), en la mayoría no ocurre esto (Asendorpf et al., 2001; Bohem et al., 2002; Kinnunen et al., 2012; Van Leeuwen et al., 2004). 
Hasta hoy, la razón de la heterogeneidad en el tamaño de los prototipos se puede adjudicar vagamente a las características muestrales. En cualquier caso, no solo el tamaño sino el número de prototipos recuperados se suman a los resultados que a lo largo de más de 20 años han favorecido el argumento de que es prematuro generalizar prototipos en múltiples culturas. Y, frente a esto, estudios muy recientes apoyan tanto la solución "tradicional" de tres conglomerados (Alessandri et al., 2014) como la de cuatro (Isler et al., 2016).

En cuanto a la distribución diferencial de mujeres y hombres en los prototipos, no se sabe aún el porqué de la misma; lo que probablemente se debe a la heterogeneidad de los resultados (Herzberg, 2009). En este trabajo, el único resultado consistente fue que las mujeres estaban sobrerrepresentadas en el prototipo No-Resiliente en ambos grupos; y que para la edad no se encontraron resultados consistentes.

Un resultado que llama la atención en este estudio es el pequeño tamaño del prototipo Resiliente en el GM. Conviene recordar que en este grupo se extrajeron soluciones desde dos hasta cinco conglomerados (datos no presentados), pero en las soluciones de cuatro y cinco, el prototipo Resiliente seguía siendo formado por los mismos individuos. Además, los conglomerados cuatro y cinco presentaban una configuración con puntajes altos o bajos en todos los rasgos, lo que dificulta una interpretación clara. En cualquier caso, pretender explicar el porqué de hallazgos como el bajo número de Resilientes comparado con el alto número de No-Resilientes, así como el haber encontrado el prototipo Disciplinado, es una tarea compleja ante la ausencia de trabajos semejantes. Sin embargo, para la cultura mexicana existe una teoría etnopsicológica de la personalidad con evidencias empíricas de tipos de personalidad (Díaz-Guerrero, 2012) que parece conveniente considerar ante estos resultados.

Con respecto a esto, Díaz-Guerrero (2012) menciona ocho tipos de personalidad producto de una cultura con un patrón formativo firme, autoritario y con una confusión importante entre el amor y el poder (Díaz-Guerrero, 2012, p.76). Cuatro de ellos, dice, describen a la gran mayoría de los mexicanos, según las evidencias recolectadas a lo largo de más de 25 años: el pasivo y obediente-afiliativo, el rebelde activamente autoafirmativo, el de control interno activo y el de control externo pasivo.

Al comparar las características atribuidas a estos tipos mexicanos con los prototipos propuestos con un modelo universalista, como es el de los CGFP (Costa \& McCrae, 2008), puede decirse que el tipo pasivo y obediente-afiliativo, descrito como ordenado, disciplinado, metódico, que planea sus cosas y es paciente, cauto y con poca impulsividad, espontaneidad y excitabilidad, puede asemejarse a las características del prototipo denominado en este estudio como Disciplinado; particularmente por que en este ultimo la característica central es la autodisciplina o el autocontrol.

De igual forma, el tipo rebelde activamente autoafirmativo muestra características de mayor agresividad, dominancia, impulsividad, problemas emocionales, ansiedad y problemas de adaptación, puede asemejarse al prototipo No-Resiliente. Mientras que el tipo control interno activo, al que se refiere como el menos común de los tipos mexicanos, se caracteriza por ser individuos cumplidos, ordenados, poco agresivos, y, sobre todo, que exhiben más recursos internos para afrontar los problemas, puede asemejarse con el prototipo Resiliente. Adicionalmente, Díaz-Guerrero (2012) indica que el tipo control externo pasivo se asemeja al rebelde activamente autoafirmativo, pero con menores habilidades intelectuales y académicas; con nuestros datos no es posible diferenciar a este tipo.

Con respecto a las comparaciones en la salud subjetiva por prototipos de personalidad, la salud subjetiva se evaluó por medio de la pregunta "¿padece algún problema de salud?", y con los puntajes del GHQ-12 como medida de la salud o distrés psicológico. En este aspecto, no se encontraron diferencias importantes entre prototipos para el GJ en respuesta a la pregunta sobre su salud, ya que la mayoría contestó no tener problemas, como sería de esperar teniendo en cuenta su edad. Sin embargo, en el GM se encontró que el prototipo No-Resiliente presentaba más problemas de salud que el Resiliente. Y en cuanto al GHQ-12, las diferencias fueron significativas tanto para los ítems positivos como para los negativos, aunque fueron más claras para los positivos en ambos grupos. De este modo, se confirma que los puntajes del Resiliente en ambos sexos están asociados con una mejor salud subjetiva, mientras que la configuración opuesta del No-Resiliente (o Sobrecontrolado) (Chapman \& Goldberg, 2011), corresponde a la peor salud. Para evitar confusiones, es importante aclarar que Kinnunen et al. (2012) nombraron como Sobrecontrolado al prototipo con configuración semejante al del prototipo aquí denominado No-Resiliente.

Finalmente, se concluye que la investigación centrada en la persona es un importante complemento a la investigación de las diferencias individuales; y, hasta donde se sabe, el presente es el primer estudio de prototipos de personalidad en Latinoamérica, lo que dificulta la comparación de los prototipos obtenidos en este estudio. Sin embargo, el hecho de haber recuperado los prototipos Resiliente y $\mathrm{No}$ Resiliente en dos grupos de edad ofrece confianza en su consistencia; y más interesante resulta haber encontrado el prototipo Disciplinado, pero se requieren más estudios para confirmarlo. 
Una limitante del estudio fue no haber incluido medidas objetivas de la salud - como masa corporal o presión sanguínea, entre otras - pero no se contaba con los recursos para hacerlo. Sin embargo, la investigación centrada en la persona favorece el desarrollo de modelos útiles para relacionar o predecir comportamientos en la vida real, tales como el estado de salud de los individuos. Así, el hecho de que los prototipos mostraran diferencias en la salud subjetiva puede ser conocimiento útil a considerar en las acciones necesarias para prevenir las enfermedades no transmisibles que afecten adversamente a la población latinoamericana.

\section{REFERENCIAS}

Akse, J., Hale, W. W., Engels, R. C., Raaijmakers, Q. A., \& Meeus, W. H. (2004). Personality, perceived parental rejection and problem behavior in adolescence. Social Psychiatry and Psychiatric Epidemiology, 39, 980-988.

Akse, J., Hale, W. W., Engels, R. C., Raaijmakers, Q. A., \& Meeus, W. H. (2007). Co-occurrence of depression and delinquency in personality types. European Journal of Personality, 21, 235-256.

Alessandri, G., Vecchione, M., Donnellan, B. M., Eisenberg, N., Caprara G. V., \& Cieciuch, J. (2014). On the crosscultural replicability of the resilient, undercontrolled, and overcontrolled personality types. Journal of Personality, 82(4), 340-353.

Asendorpf, J. B., Borkenau, P., Ostendorpf, F., \& van Aken, M. A. (2001). Carving personality description at its joints: Confirmation of three replicable personality prototypes for both child and adults. European Journal of Personality, 15, 169-198. doi: 10.1002/per.408.

Avdeyeva, T. V., \& Church, A. T. (2005). The cross-cultural generalizability of personality types: A Philippine study. European Journal of Personality, 19, 475-499.

Barbaranelli, C. (2002). Evaluating cluster analysis solutions: An application to the Italian NEO Personality Inventory. European Journal of Personality, 16, s43-s55.

Boehm, B., Asendorpf, J. B., \& Avia, M. D. (2002). Replicable types and subtypes of personality: Spanish NEO-PI samples. European Journal of Personality, 16, s25-s41.

Borkenau, P., Hrebícková, M., Kuppens, P., Realo, A., \& Allik, J. (2013). Sex differences in variability in personality: A study in four samples. Journal of Personality, 81(1), 49-60. doi: 10.1111/j.1467-6494.2012.00784.x.

Caspi, A. (1998). Personality development accross the life course. En W. Damon \& N. Eisenberg (Eds.), Handbook of child psychology: Vol. 3. Social, emotional, and personality development (pp. 311-388). New York: Wiley.

Chapman, B. P., \& Goldberg, L. G. (2011). Replicability and 40-year predictive power of childhood ARC types. Journal of Personality and Social Psychology, 101(3), 593-606. doi: $10.1037 / \mathrm{a} 0024289$.

Costa, P. T. Jr., \& McCrae, R. (2008). Inventario de personalidad NEO Revisado (NEO PI-R). Inventario NEO Reducido de Cinco Factores (NEO-FFI). Manual Profesional (3ra. edición revisada y ampliada). Madrid, España: TEA Ediciones S. A.

Church, A. T. (2016). Personality traits across cultures. Current Opinion in Psychology, 8, 22-30.

De Fruyt, F., Mervielde, I., \& Van Leeuwen, K. (2002). The consistency of personality type classification across samples and five-factor measures. European Journal of Personality, 16, s57-s72. doi: 10.1002/per.444.

Díaz-Guerrero, R. (2012). Psicología del Mexicano. Descubrimiento de la etnopsicología (6ta. ed., reimpresión). México, D. F.: Trillas.

Donnellan, M. B., \& Robins, R. W. (2010). Resilient, overcontrolled, and undercontrolled personality types: Issues and controversies. Social and Personality Psychology Compass 3, 1-14. doi: 10.1111/j.1751-9004.2010.00313.x.

Ekehammar, B., \& Akrami, N. (2003). The relation between personality and prejudice: A variable- and a person-centered approach. European Journal of Personality, 17, 449464. doi: 10.1002/per.494.

García, O. Aluja, A., \& García, L. F. (2004). Psychometric properties of the Goldberg's 50 Personality Markers for the Big Five Model: A study in Spanish language. European Journal of Psychological Assessment, 20(4), 310-319.

Goldberg, D. P., \& Williams, P. (1988). A user's guide to the General Health Questionnaire. Windsor, UK: NFER-Nelson.

González, S., Tello, J., Silva, P., Lüders, C., Butelmann, S., Fristch, R., Solar, F., et al. (2012). Calidad de vida en pacientes con discapacidad motora según factores sociodemográficos y salud mental. Revista Chilena de NeuroPsiquiatría, 50(1), 23-34.

Hankins, M. (2008). The reliability of the twelve-item General Health Questionnaire (GHQ-12) under realistic assumptions. BMC Public Health, 8, 355.

Hart, D., Burock, D., London, B., Atkins, R., \& Bonilla-Santiago, G. (2005). The relation of personality types to physiological, behavioural, and cognitive processes. European Journal of Personality, 19, 391-407.

Hernández, R., Fernández, C., \& Baptista, P. (2010). Metodología de la investigación. (5ta. ed.). Peru: McGrawHill.

Herzberg, P. Y. (2009). Beyond "accident-proneness": Using five-factor model prototypes to predict driving behavior. Journal of Research in Personality, 43, 1096-1100. doi: 10.1016/j.jrp.2009.08.008.

Herzberg, P. Y., \& Roth, M. (2006). Beyond resilients, undercontrollers, and overcontrollers? An extension of personal- 
ity prototype research. European Journal of Personality, 20, 5-28. doi: 10.1002/per.557.

Hoyle, R. H. (2010). Personality and self-regulation. En Rick H. Hoyle (Ed.), Handbook of personality and self-regulation (pp. 1-18). West Sussex, UK: Wiley-Blackwell.

Isler, L., Liu, J. H., Sibley, C. G., \& Fletcher, G. J. O. (2016). Self-Regulation and personality profiles: Empirical development, longitudinal stability and predictive ability. European Journal of Personality, 30(3), 274-287. doi: 10.1002/ per.2054.

Kaleta, D., Polańska, K., Dziankowska-Zaborszcsyk, E., Hanke, W., \& Drygas, W. (2009). Factors influencing self-perception of health status. Central European Journal of Public Health, 17(3), 122-127.

Kinnunen, M. L., Metsäpelto, R. L., Feldt, T., Kokko, K., Tolvanen, A., Kinnunen, U., Leppänen, E., et al. (2012). Personality profiles and health: Longitudinal evidence among Finnish adults. Scandinavian Journal of Psychology 53, 512-522. doi: http://dx.doi.org/10.1111/j.14679450.2012.00969.x.

Klimstra, T. A., Hale, W. W., Raaijmakers, Q. A., Branje, S. J., \& Meeus, W. H. (2010). A developmental typology of adolescent personality. European Journal of Personality, 24, 309-323.

Lahey, B. B. (2009). Public health significance of neuroticism. American Psychologist, 64, 241-256.

Martínez, P., \& Cassaretto, M. (2011). Validación del Inventario de los Cinco Factores NEO-FFI en español en estudiantes universitarios peruanos. Revista Mexicana de Psicología, 28, 63-74.

Meda-Lara, R. M., Moreno-Jiménez, B., García, L. F., Palomera Chávez, A., \& Mariscal de Santiago, M. V. (2015). Validez factorial del NEO-FFI en una muestra mexicana: Propuesta de una versión reducida. Revista Mexicana de Psicología, 32(1), 57-67.

McDowell, I. (2006). Measuring health - a guide to rating scales and questionnaires ( $3^{\mathrm{a}}$.ed.). New York: Oxford Press.

Miller, J. D. (2012). Five-factor model personality disorder prototypes: A review of their development, validity, and comparison to alternative approaches. Journal of Personality, 80(6), 1565-1591.

Roberts, B. W., Kuncel, N., Shiner, R., Caspi, A., \& Goldberg, L. R. (2007). The power of personality: The comparative validity of personality traits, socioeconomic status, and cognitive ability for predicting important life outcomes. Perspectives on Psychological Science, 2, 313-345.

Robins, R. W., John, O. P., Caspi, A., Moffitt, T. E., \& Stouthamer-Loeber, M. (1996). Resilient, overcontrolled, and undercontrolled boys: Three replicable personality types. Journal of Personality and Social Psychology, 70, 157-171.

Roth, M., \& von Collani, G. (2007). A head-to-head comparison of big five types and traits in the prediction of social attitudes: Further evidence for a five-cluster typology. Journal of Individual Differences, 28, 138-149.

Sánchez-López, M. P., \& Dresch, V. (2008). The 12-item General Health Questionnaire (GHQ-12): Reliability, external validity and factor structure in the Spanish population. Psicothema, 20(4), 839-843.

Smith, T. W., Williams, P. G., \& Segerstrom, S. C. (2015). Personality and physical health. En M. Mikulincer \& P. R. Shaver (Editors-in-Chief), Handbook of personality and social psychology: Vol. 4. Personality processes and individual differences (pp. 639-661. Washington: American Psychological Association. doi: http://dx.doi.org/10.1037/14343029.

Smith, A. B., Oluboyede, Y., West, R., Hewison, J., \& House, A. O. (2013). The factor structure of the GHQ-12: the interaction between item phrasing, variance and levels of distress. Quality of Life Research, 22, 145-152.

Sociedad Mexicana de Psicología. (2007). Código Ético del Psicólogo. México: Trillas.

Solís-Cámara, P., Meda Lara, R. M., Moreno-Jiménez, B., \& Juárez, P. (2016). Estructura factorial del Cuestionario de Salud General GHQ-12 en población general de México. Salud \& Sociedad, 7(1), 62-76.

Steca, P., Alessandri, G., \& Caprara, G. V. (2010). The utility of a well-known personality typology in studying successful aging: Resilients, undercontrollers, and overcontrollers in old age. Personality and Individual Differences, 48, 442446.

Steinley, D. (2003). Local optima in K-means clustering: What you don't know may hurt you. Psychological Methods, 8 , 294-304.

Strus, W., Cieciuch, J., \& Rowinski, T. (2014). The circumplex of personality metatraits: A synthesizing model of personality based on the big five. Review of General Psychology 18, (4), 273-286. doi: http://dx.doi.org/10.1037/gpr0000017.

Tuuliainen/Kirsi Sipilä, L., Mäki, P., Könönen, M., \& Suominen, A. L. (2015). Association between clinical signs of temporomandibular disorders and psychological distress among an adult Finnish population. Journal of Oral \& Facial Pain and Headache, 29(4), 370-377. doi: 10.11607/ ofph.1439.

Urzúa, A., Caqueo-Urízar, A., Bargsted, M., \& Irarrázaval, M. (2015). ¿Afecta la forma de puntuación la estructura factorial de GHQ-12? Cadernos de Saúde Pública, 31(6), 13051312.

Van Leeuwen, K., De Fruyt, F., \& Mervielde, I. (2004). A longitudinal study of the utility of the resilient, overcontrolled, and undercontrolled personality types as predictors of children's and adolescents' problem behavior. International Journal of Behavioral Development, 28, 210-220. Recuperado de http://www.tandf.co.uk/journals/pp/01650254.html.

Weir, R. C., \& Gjerde, P. F. (2002). Preschool personality prototypes: Internal coherence, cross-study replicability, and 
developmental outcomes in adolescence. Personality and Social Psychology Bulletin, 28, 1229-1241.

Xie, X., Chen,W., Lei, L., Xing, C., \& Zhang, Y. (2016). The relationship between personality types and prosocial behavior and aggression in Chinese adolescents. Personality and Individual Differences, 95, 56-61.
Zawadzki, B., \& Strelau, J. (2003). Trzy podstawowe typy czy cztery struktury temperamentu? [Three basic types or four structures of temperament?]. Czasopismo Psychologiczne, 6, 271-285. 